\title{
The B-ADENOMA Study: Bowelscope - Accuracy of Detection using Endocuff Optimisation of Mucosal Abnormalities: Study Protocol for randomised controlled trial
}

\section{(ㄷ)(1) $(2) \Theta$}

\section{Authors}

Wee Sing Ngu ${ }^{1}$, Martin Walls ${ }^{1}$, Pradeep Bhandari ${ }^{2}$, Clive Stokes ${ }^{3}$, Nikki Totton ${ }^{4}$, Zoe Hoare ${ }^{4}$, Lexi Bastable ${ }^{4}$, Colin Rees $^{1}$

Institutions

1 South Tyneside NHS Foundation Trust - Gastroenterology, South Shields, Tyne and Wear, United Kingdom of Great Britain and Northern Ireland

2 Queen Alexandra Hospital - Gastroenterology, Portsmouth, Portsmouth, United Kingdom of Great Britain and Northern Ireland

3 Gloucestershire Hospitals NHS Foundation Trust - BOSS trials office, Gloucester, Gloucestershire, United Kingdom of Great Britain and Northern Ireland

4 North Wales Organisation for Randomised Trials in Health - Statistics, Bangor, United Kingdom of Great Britain and Northern Ireland

submitted 16.11.2017

accepted after revision 26.2.2018

Bibliography

DOI https://doi.org/10.1055/a-0591-9308 |

Endoscopy International Open 2018; 06: E872-E877

(c) Georg Thieme Verlag KG Stuttgart · New York

ISSN 2364-3722

Corresponding author

Martin Walls, South Tyneside NHS Foundation Trust Gastroenterology, South Tyneside District Hospital, Harton Lane South Shields NE34 OPL, United Kingdom of Great Britain and Northern Ireland

Fax: + 01914041000

martin.walls@doctors.org.uk

martin.walls@stft.nhs.uk

\section{ABSTRACT}

Background and study aims UK Bowel Cancer Screening flexible sigmoidoscopy (BowelScope) currently offers patients aged 55 a one-off flexible sigmoidoscopy for adenoma clearance to decrease colorectal cancer incidence by in- terrupting the adenoma-carcinoma sequence. Recent evidence has shown maximum benefit in increasing adenoma detection rate (ADR) using the Endocuff Vision device in the left side of the colon and in screening patients. Currently, ADR is low and shows unacceptable variation in BowelScope. ADR is a quality indicator in screening sigmoidoscopy and higher rates have been shown to reduce colorectal cancer incidence.

Patients and methods This will be a prospective, multicenter, UK-based randomized controlled trial (RCT) comparing ADR in Endocuff-assisted versus standard bowel cancer screening flexible sigmoidoscopy (BowelScope). All patients aged 55 to 61 years invited to BowelScope screening and able to give informed consent will be eligible for recruitment. Exclusion criteria include absolute contraindications to flexible sigmoidoscopy, known or suspected large bowel obstruction or pseudo-obstruction, colonic strictures or polyposis syndromes, known severe diverticular segment, active colitis, inability to give informed consent, anticoagulation precluding polypectomy and pregnancy. Patients will be randomized on the day of procedure to Endocuff-assisted flexible sigmoidoscopy or standard flexible sigmoidoscopy, stratified by age group and sex. Baseline, endoscopy and polyp data were collected as well as nurse and patient assessment of comfort. Polyp histology was collected when available. Patients will be asked to return a comfort questionnaire the following day and were followed up for 14 days for complications.

The study will take place across 12 to 20 hospital trusts across the UK and recruited 3222 patients.

Results The ADENOMA trial will be designed to demonstrate a significant improvement in ADR with maximal effect in the left colon and in fecal occult blood test-positive screening patients. This trial will be the first RCT to look at Endocuff Vision in bowel cancer screening flexible sigmoidoscopy. We will aim to establish whether Endocuff vision improves ADR in this population.

Clinicaltrials.gov Identifier: NCT03072472 


\section{Introduction}

Colorectal cancer (CRC) is the fourth most common cancer in the UK with 40,000 new cases diagnosed annually [1]. Most CRCs arise from the adenoma-carcinoma sequence which is a process that can take up to 10 years [2]. Patients diagnosed at the earliest stages of the disease have a 5-year survival rate of $93.2 \%$ compared to $6.6 \%$ during the advanced stages of the disease [3]. Population screening programs allow for earlier detection and removal of adenomas that may become malignant over time, improving outcomes following CRC diagnosis and reducing CRC mortality [4]. Following a large trial of one-off flexible sigmoidoscopy and adenoma clearance as a screening procedure across 14 UK centers in 170,432 patients demonstrating a $50 \%$ reduction in incidence of distal and sigmoid cancers [5], this procedure was rolled out nationally as the English Bowel Scope Screening (BSS) program in 2013. BSS invites adults aged 55, with 56- to 60-years-olds able to opt-in, for a one-off flexible sigmoidoscopy on the basis that adenoma clearance in adults aged 55 to 64 years reduces CRC incidence by $23 \%$ and mortality by $31 \%$ in intention to treat analysis [5].

Adenoma detection rate (ADR) is the most important marker of mucosal visualization and a surrogate marker of high-quality colonoscopy [6]. A $1 \%$ increase in ADR is associated with a $3 \%$ decrease in interval colorectal cancer [7]. In the NHS BCSP, ADR has been reported at $29 \%$ in women and $43 \%$ in men undergoing colonoscopy after positive fecal occult blood testing [8]. In the BSS program of one-off flexible sigmoidoscopy, national ADR is comparatively lower at $8.8 \%$ (Claire Nickerson, personal communication, March 21, 2016) with a wide variation of $7 \%$ to $12 \%$ between endoscopists [9]. Similar flexible sigmoidoscopy screening programs in Europe have demonstrated ADRs of $15 \%$ [10] and 18\% [11] although the reasons for that are likely to be multifactorial relating to differing populations. Another marker that is often used is adenoma miss rates, which also demonstrate a wide variation of $6 \%$ to $27 \%$ in clinical practice $[12,13]$. Reasons for lesions not being detected at flexible sigmoidoscopy can be extrapolated from colonoscopy data and include suboptimal technique; shorter withdrawal time; inadequate bowel preparation; presence of flat, depressed or subtle lesions; and inability to visualize the proximal side of haustral folds, flexures (blind spots) and rectal valves $[14,15]$.

A device such as the Endocuff Vision is likely to have the greatest benefit in the sigmoid and distal colon, where mucosal visualization is most impaired by mucosal folds. The ADENOMA study was a randomized controlled trial comparing use of Endocuff Vision-assisted colonoscopy versus standard colonoscopy [16]. There was no suggestion of adverse impact on overall patient experience or screening uptake. The trial demonstrated a significant improvement in ADR, more so in the left colon and in patients examined as part of the NHS Bowel Cancer Screening Programme (asymptomatic patients).

Bowelscope screening will only achieve its desired effect if adenoma detection is optimal. In the current program, adenoma detection rates are lower than in the flexible sigmoidoscopy trial and there is wide variation. It is imperative that ADR is im-

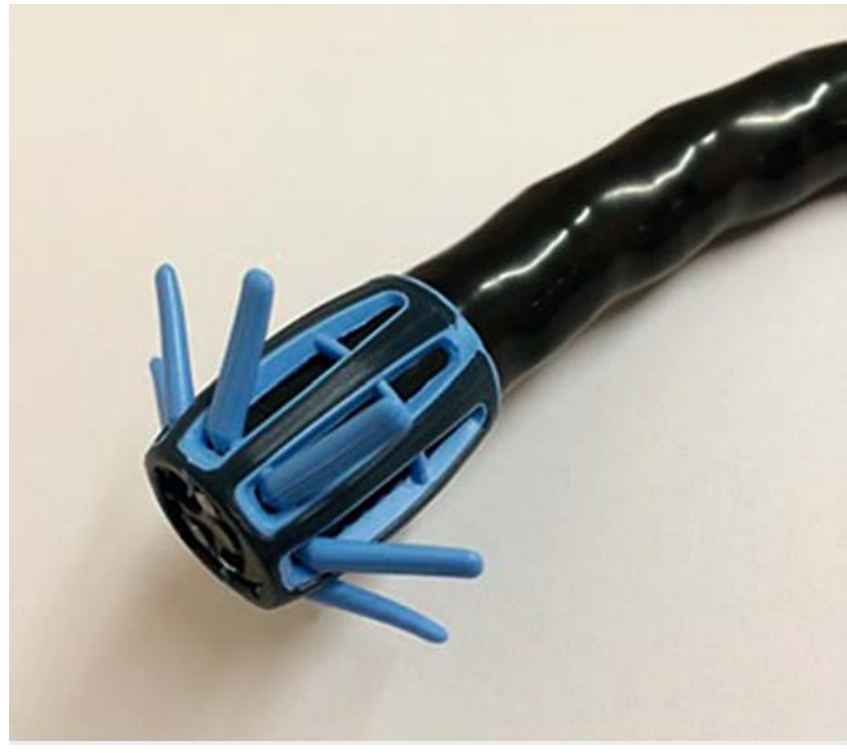

×ig. 1 Endocuff Vision.

proved to a level more consistent with cancer prevention levels demonstrated both in the original flexible sigmoidoscopy screening trial [5] and in other programs. In addition, the ADR of endoscopists with lower ADRs should be improved.

With the aid of the colonoscopic cuff Endocuff Vision, we will aim to improve visualization of the colonic mucosa by flattening colonic folds and manipulating them away from the field of forward view. This clinical randomized study will be conducted in subjects referred and scheduled for screening flexible sigmoidoscopy via the NHS English Bowel Scope Screening (BSS) Programme and compare Endocuff Vision-Assisted flexible sigmoidoscopy (EAFS) with standard flexible sigmoidoscopy (SFS).

\section{Patients and methods}

The Endocuff Vision (ARC Medical Design Ltd and Diagmed, UK) is a device (CE marked in UK) made of a soft plastic material with a unique dynamic shape ( $\triangleright$ Fig. 1 ). The core is made of polypropylene and the "finger-like" projections are made of a thermoplastic elastomer. Endocuff comes in four color-coded sizes (purple, blue, green and orange) to fit a range of pediatric and adult colonoscopes. The backwards-pointing, flexible, "finger-like" projections are spaced at intervals around the device circumference with the following dimensions in different cuff sizes (length $23.8 \mathrm{~mm} \times$ diameters $16.1 / 16.7 / 17.2 / 18.5 \mathrm{~mm}$ with the finger-like projections folded back and 39.7/39.7/ $39.7 / 39.66 \mathrm{~mm}$ with finger-like projections opened out). The device is mounted at the tip of the scope and held on by friction (pull-off force is a minimum of 10 Newtons). The ADENOMA trial reported no significant cuff-related adverse events. The Endocuff Vision does not appear to adversely affect patient comfort and may in fact enhance patient experience as mucosal views may be more readily achieved without excessive colonic distension or over-insertion of the endoscope. 
Use of the Endocuff is contraindicated in patients with known colonic strictures and active inflammatory disorders such as acute infective colitis, active (not quiescent) colonic Crohn's disease, active (not quiescent) ulcerative colitis and acute diverticulitis ( $>$ Fig. 1 ).

Endocuff Vision is placed snugly around the colonoscope tip prior to insertion. It does not project beyond the tip of the scope, providing an unrestricted view. It does not trap fecal residue. It helps anchor the scope tip against the bowel wall to provide a stable platform of access. The soft, elastic projections are pushed back (recoiled) towards the scope shaft during insertion but evert during withdrawal to hold colon folds away from the field of view.

Endocuff Vision may therefore benefit patients by resulting in more accurate examinations with lower rates of missed polyps. It may also benefit NHS and other healthcare providers because of reduced risk of CRC, with fewer polyp "misses," and improved ADRs, with a potential correlating reduction in interval cancer rates

\section{Study objectives}

The primary study objective will be to detect a difference in ADR between EAFS and SFS. The secondary objectives will be to: 1) detect a difference in mean adenomas detected per procedure (MAP ${ }^{2}$ ) between EAFS and SFS; 2 ) establish the rate of cuff exchange (that is, how often the cuff has to be removed); 3) demonstrate non-inferiority in complete withdrawal time in procedures where no polyps are detected between EAFS and SFS; 4) compare overall procedure time between EAFS and SFS; 5) measure differences in ADR accounting for patient procedure-based variables (e. g. accounting for extent of examination and bowel preparation); 6) compare the rate of discovered cancers between EAFS and SFS; 7) demonstrate non-inferiority for examination extent for EAFS compared to SFS; 8) demonstrate non-inferiority of patient satisfaction with EAFS compared to SFS; 9) identify any difference in future colonoscopic workload produced by increased ADR in terms of number of patients referred for full colonoscopy between the EAFS and SFS groups; 10) compare the ADR of the first $20 \%$ of patients scoped by each colonoscopist with the last $20 \%$ of patients in each arm to identify any changes in ADR to assess any learning curve effect; and 11) compare the baseline ADR of each colonoscopist prior to trial recruitment with their individual ADR in patients where Endocuff ${ }^{\mathrm{TM}}$ Vision was not used.

\section{Study design}

This will be a clinical, randomized, multicenter study designed to compare EAFS and SFS in patients participating in the national flexible sigmoidoscopy screening (Bowelscope) programme. Patients will be recruited from 12 to 20 sites around England. Recruitment will begin initially in South Tyneside District Hospital and University Hospital North Tees as part of an "advance test" for no longer than 1 month, to allow for testing of the protocol and data collection process. Any required amendments will then be made before rolling out to the other recruiting sites. To adequately power to detect a $3 \%$ increase in ADR we will recruit 3222 patients. These patients will have been re- ferred for screening flexible sigmoidoscopy at one of the participating sites. All potential participants will be given a study patient information leaflet in advance of their procedure, allowing adequate time to read the information leaflet before consenting to the study. On attending the endoscopy unit for their procedure, they will be approached by a member of the research team and given the opportunity to discuss the study. If they are willing to proceed with the study, they will complete written consent forms, and baseline data will be collected. Patients will then be randomized to either EAFS or SFS using a secure, webbased platform [17].

The procedure will be performed and intra-procedure data collected in a case report form (CRF) by a member of the research team. Any polyps detected and removed will be followed up, and histological diagnosis recorded post-procedure by the research team. All flexible sigmoidoscopes will be calibrated and serviced according to local guidelines. All polyps will be recorded regardless of whether they are removed or not for calculation of MAP2 rates. It is standard practice to use no sedative or otherwise cognitively impairing medications, thus allowing immediate assessment of patient experience post-procedure.

Patient study participation will be 14 days to allow collection of standard post-flexible sigmoidoscopy complication and data on serious adverse events (SAEs). This is the standard follow up for the Bowel Scope program and was not extended so that trial data are directly comparable with other procedure data. There will be no need for additional follow-up visits as a result of participation. The unit's standard care for patients, including timing of outpatient appointments and results, will not be affected. Data will be collated and analyzed by the research team. Adverse events (AEs) will be collated by the research team, the Trial Manager will discuss them with independent clinicians and report to the Data Monitoring Committee, which will assess the relationship of the AEs to use of the Endocuff Vision device. The results of this discussion will also be presented to the Trial Steering Committee. There are no known complications or AEs associated with Endocuff Vision.

\section{Data collection}

Before the procedure, data will be collected on patient demographics (age, gender), past abdominal surgical history, and number of enemas used pre-procedure. During the procedure, data will be collected on polyps detected (total number, plus for each polyp seen, location, size, morphology, removed [Y/ $\mathrm{N}$ ], removal method); extent of examination (anatomical location and distance in centimeters); insertion time; withdrawal time; quality of bowel preparation; use of carbon dioxide insufflation; presence of diverticulosis; patient satisfaction and comfort scores; and immediate complications. Post-procedure, data will be collected on polyp histology, complications up to 14 days, AEs, and patient comfort post-procedure and postprocedure Day 1. 


\section{Inclusion, exclusion and withdrawal criteria and indications for cuff removal}

Patients will be included if they are age 55 to 61 years, have been referred for screening flexible sigmoidoscopy, and are able to give informed consent. They will be excluded if they have absolute contraindications to flexible sigmoidoscopy; established or suspicion of large bowel obstruction or pseudo-obstruction; known colon cancer or polyposis syndromes; known colonic strictures; known severe diverticular segment (that is likely to impede sigmoidoscope passage); have known active colitis (ulcerative colitis, Crohn's colitis, diverticulitis, infective colitis); lack the capacity to give informed consent; are on clopidogrel, warfarin, or other new generation anticoagulants that have not been stopped for the procedure, as these will preclude polyp removal; or are pregnant.

Patients with a new diagnosis of polyposis syndrome will be withdrawn from the trial.

Indications for cuff removal are acute angulation in a fixed sigmoid colon, rendering scope insertion not feasible with the Endocuff Vision mounted; new diagnosis of active colitis (where the endoscopist is concerned regarding the risk of mucosal damage); identification of a colonic stricture; and new cancer diagnosis if progression of the colonoscope with the Endocuff Vision attached is not possible.

\section{Endoscopists and training with Endocuff Vision}

Trial recruitment will involve endoscopists with a wide range of experience, however, all will have been accredited to undertake BowelScope screening. All endoscopists at participating units will undergo theoretical and practical sessions of training (using DVD tutorials) with Endocuff Vision and will have a lifetime experience of at least 10 cases (flexible sigmoidoscopy or colonoscopy) with the device prior to study commencement.

\section{Adverse events}

Risks of AEs for the EAFS, including bleeding and perforation, are believed to be equivalent to SFS. AE rates will be measured and recorded in the patients' medical notes and on case report forms. AEs are defined as any new medical occurrence or worsening of a preexisting medical condition in a patient. All AEs will be grouped according the BCSP Adverse Incident criteria and will be assessed by an Investigator to define the relationship to the Endocuff Vision. All SAEs will be treated clinically as appropriate and reported to the trial team as soon as the research team becomes aware of the event using the studyspecific SAE Form, in line with current GCP guidance. SAEs will be assessed for expectedness, severity and relatedness to the Endocuff Vision device. Any related and unexpected SAEs will be reported to the main NHS research ethics committee within 15 days of the trial team becoming aware of the events, using the National Research Ethics Service SAE form for non-CTIMPs. An event will be considered to be serious if it results in death; is life-threatening; results in hospitalization or prolongation of existing hospitalization (except routine planned admission, including admission for flexible sigmoidoscopy procedures as part of this study); leads to persistent significant disability or incapacity; or is otherwise considered to be medically significant by the investigator.

AEs and SAEs will be recorded for the 14-day period from the day of flexible sigmoidoscopy or until withdrawal. SAEs will be followed until resolution, death, or until resolution with sequelae. In addition, SAEs must be recorded in the Case Report Form in the Adverse Events section. SAEs must be reported even if they are considered to be expected events or unrelated events by the Investigator.

\section{Assessment and follow-up}

Clinical follow-up will be according to local unit policy. Flexible sigmoidoscopy-related complications are routinely recorded up to 14 days post-procedure, in keeping with UK NHS Bowel Scope operating procedures. All patients with polyps who require full colonoscopy (according to BCSP guidelines [18]) will have this recorded in the Case Report Form. BSS defines all procedures as complete if the procedure is commenced, therefore, the extent and limiting factor of the procedure will be recorded. Eligible, consented patients will remain in the study for 14 days following flexible sigmoidoscopy. All AEs will also be reported for the 14-day period post-flexible sigmoidoscopy for all patients in the study. We will review complication data and AEs at 14 days by the most appropriate method for the population at each local site. This will consist of either a phone call to the patient or review of medical notes and hospital databases. If we are aware that a patient presents to a different hospital post-procedure than the hospital where the flexible sigmoidoscopy was performed, we will contact the individual's general practitioner to obtain information regarding the event. No additional visits will be required for patients who enter the study. Any follow-up appointments post-flexible sigmoidoscopy will be as per routine care for the respective unit. The timescale for the outpatient appointment and subsequent care will be unaltered by participation in the study.

\section{Data management}

All data will be entered and stored in MACRO [19], an electronic data collection system that is backed up to a central server. This will allow the data to be entered, reviewed and amended while keeping an audit log. The MACRO system is designed and programmed with controls in place to minimize any data entry errors. Consent forms will be stored for 15 years and trial electronic data and all other appropriate documentation stored for 5 years after the completion of the study, including the followup period, as per CTU policy.

\section{Sample size}

The B-ADENOMA study has been powered to detect a $3 \%$ absolute difference in the primary outcome, ADR, as that has been evaluated as a clinically important difference. This would increase the ADR from $8.8 \%$, as currently found (oral communication, NHS Bowel Cancer Screening Programme national office, November 1, 2016), to $11.8 \%$. Using a two-sided proportional test with a $5 \%$ significance level and $80 \%$ power, the trial will require 1611 patients per group (3222 overall). 


\section{Data analysis}

ADR will be compared between the EAFS and FSF groups using chi-squared tests and logistic regression models to take into account any factors that are considered important within the data, i.e. stratification variables. Secondary outcomes will be analyzed for non-inferiority with $95 \%$ confidence intervals (withdrawal and complete procedure time, patient satisfaction and examination extent). The number of adenomas detected per is expected to have a positively skewed distribution and so normality testing and appropriate analysis will be completed following the outcome to compare between the EAFS and FSF groups

ADR will be analyzed to ensure patient procedure-based variables, for example, extent of examination and bowel prep, to not introduce bias by artificially reducing the ability to detect adenomas.

Rates of cuff exchange and cancer detection will be calculated, along with $95 \%$ confidence intervals for those allocated to the EAFS group. To assess the difference between the EAFS and SFS groups in proportion of patients who require a follow-up procedure, analysis will be completed in line with that completed on the primary outcome.

To identify changes in ADR over time of the study, the detection rate in the first $20 \%$ of patients per endoscopist will be compared with the detection rate in th last $20 \%$ of their patients. In addition, the ADR for each endoscopist prior to the trial will be compared with their ADR during the trial in cases where Endocuff Vision is not used.

All analyses will be completed on an intention-to-treat basis. Any non-inferiority analysis will additionally be completed on a per protocol basis, which will be considered as a sensitivity analysis for the original results.

\section{Ethical considerations}

Ethical approval has been granted via the NHS Research Ethics Committee (REC) prior to the study start, with approval already given by the Bowel Cancer Screening Programme Research Committee. There are no known additional risks to patients associated with use of the Endocuff Vision device. Adding the Endocuff Vision will not add significantly to the duration of the procedure, although if the ADR increases significantly, the procedure may take longer due to increased time to undertake polypectomy. Patients will be informed of the risks associated with standard flexible sigmoidoscopy and consented for the procedures as per standard clinical practice in each center, in addition to a study-specific consent form, which will have been discussed with each patient by the research team. Consent to enter the study will be sought from each participant only after a full explanation has been given, an information leaflet offered, and time allowed for consideration. Signed participant consent will be obtained. Declining participation will have no impact on further treatment or care. The study will be conducted in accordance with the recommendations for physicians involved in research on human subjects adopted by the 18th World Medical Assembly, Helsinki 1964 and later revisions.
Confidentiality will be maintained in line with the Data Protection act under the overall responsibility of the Chief Investigators.

\section{Sponsor and funding}

South Tyneside Foundation Trust will be the sponsor for this trial. This study will be conducted using existing BCSP lists. Funding will be provided by ARC Medical Design Ltd to cover the Endocuff Vision devices. Clinical trial unit costs, results analysis and other trial costs are independent of this funding and will be met by portfolio adoption to ensure that NHS support costs are available to support consent and data collection. Principal investigators will be supported by a research team and they will invite, assess, and consent participants, and collect data.

\section{Data monitoring}

The Trial Steering Committee will meet every 6 months during the study period. This group will comprise a chair, one independent clinician, one independent statistician and a patient/service user representative. An independent data monitoring committee will be convened and will meet every 4 months from the start of the trial.

The study will be monitored by a clinical trial team and they must be allowed to visit all study site locations periodically to review the quality of the data and the integrity of the study, beginning with an initial visit after recruitment of approximately 20 to 50 patients. The trial manager will perform routine checks of MACRO data entry to ensure validity of the data entered remotely by sites. Full source data verification will occur for a proportion of patients who enter the trial, chosen at random. These patients' trial packs, inclusive of Case Report Forms, will be compared with the medical notes and MACRO entries. Data from the CRF will be uploaded onto the electronic CRF system by the research team thereafter. These data will be anonymized, with only a study number being recorded. At monitoring visits, the trial manager will discuss the conduct of the study with the study team and review the Investigator Site File. CTU staff will provide advice and oversight for this process. In addition, the study may be evaluated by an auditor or regulatory government inspector, who must also be allowed access to all case report forms, source documents, study files and study facilities. Further visits will be triggered by high screening/low recruitment rates or concerns regarding data quality. Remote monitoring through MACRO data submission will occur regularly.

\section{Dissemination}

B-ADENOMA has been reviewed and supported by the National Cancer Research Institute Colorectal Cancer Screening and Prevention subcommittee and the British Society of Gastroenterology (BSG). The BSG is committed to disseminating the study findings. Statistical analysis will be performed by the clinical trials unit under the supervision of Professor Colin Rees and the results will be written up in the form of a report by the research and academic team. Findings will be presented to the Trial Steering Group (TSG). Data from the study will be dissemi- 
nated to participating patients within the study via the Trust's websites. The results will then be submitted for publication in international peer-reviewed journals and presented to the BSG Endoscopy Research Committee, the Bowel Cancer Screening Programme and learned scientific societies. Feedback will be given to regional and national endoscopy leads, maximizing the exposure of findings to endoscopists. Data will also be disseminated to local networks, and at national and international symposia.

\section{Competing interests}

Colin J Rees: Research funding: ARC medical, Norgine, Olympus Medical; Paid legal expert: ARC medical. Pradeep Bhandari: Research funding: Norgine, Pentax, Fujifilm, 3D Matrix; Educational Grant: Olympus.

\section{References}

[1] Cancer Research UK. Bowel Cancer Incidence Statistics. 2013: Available from: http://www.cancerresearchuk.org/health-professional/ cancer-statistics/statistics-by-cancer-type/bowel-cancer/incidence\#ref-0 [cited 2016 Mar 4].

[2] Leslie A, Carey FA, Pratt NR et al. The colorectal adenoma-carcinoma sequence. $\mathrm{Br}$ J Surg 2002; 89: 845-860

[3] National Cancer Intelligence Network. Colorectal Cancer Survival by Stage. 2009: Available from: http://www.ncin.org.uk/publications/ data_briefings/colorectal_cancer_survival_by_stage [cited 2016 Mar 4].

[4] Rees CJ, Bevan R. The National Health Service Bowel Cancer Screening Program: the early years. Expert Rev Gastroenterol Hepatol 2013; 7: $421-437$

[5] Atkin WS, Edwards R, Kralj-Hans I et al. Once-only flexible sigmoidoscopy screening in prevention of colorectal cancer: a multicentre randomised controlled trial. Lancet 2010; 375: 1624-1633

[6] Munroe CA, Lee P, Copland A et al. A tandem colonoscopy study of adenoma miss rates during endoscopic training: a venture into uncharted territory. Gastrointest Endosc 2012; 75: 561- 567
[7] Corley DA, Jensen CD, Marks AR et al. Adenoma detection rate and risk of colorectal cancer and death. N Engl J Med 2014; 370: 1298 1306

[8] Logan RFA, Patnick J, Nickerson C et al. Outcomes of the Bowel Cancer Screening Programme (BCSP) in England after the first 1 million tests. Gut 2012; 61: 1439-1446

[9] Bevan R, Blanks R, Nickerson C et al. PWE-365 Exploring adr performance in the nhs bowel scope programme. Gut 2015; 64: (Suppl. 01): A371

[10] Gondal G, Grotmol T, Hofstad B et al. The Norwegian Colorectal Cancer Prevention (NORCCAP) screening study: baseline findings and implementations for clinical work-up in age groups 50-64 years. Scand J Gastroenterol 2003; 38: 635-642

[11] Segnan N, Senore C, Andreoni B et al. Baseline findings of the Italian multicenter randomized controlled trial of "once-only sigmoidoscopy"-SCORE. J Natl Cancer Inst 2002; 94: $1763-1772$

[12] Rex DK, Cutler CS, Lemmel GT et al. Colonoscopic miss rates of adenomas determined by back-to-back colonoscopies. Gastroenterology 1997; 112: $24-28$

[13] Hixson LJ, Fennerty MB, Sampliner RE et al. Prospective study of the frequency and size distribution of polyps missed by colonoscopy. J Natl Cancer Inst 1990; 82: 1769-1772

[14] Faiss S. The missed colorectal cancer problem. Dig Dis 2011; 29: (Suppl. 01): 60-63

[15] Rees CJ, Rajasekhar PT, Rutter MD et al. Quality in colonoscopy: European perspectives and practice. Expert Rev Gastroenterol Hepatol 2014; 8: 29-47

[16] Bevan R, Ngu WS, Saunders BP et al. The ADENOMA Study. Accuracy of Detection using Endocuff VisionTM Optimization of Mucosal Abnormalities: study protocol for randomized controlled trial. Endosc Int Open 2016; 4: E205-E212

[17] Russell D, Hoare ZSJ, Whitaker R et al. Generalized method for adaptive randomization in clinical trials. Stat Med 2011; 30: 922-934

[18] Public Health England. NHS public health functions agreement 2017-18 Service specification No. 26A NHS bowel scope screening programme. 2017: Available from: https://www.england.nhs.uk/ wp-content/uploads/2017/04/service-spec-26a.pdf

[19] MACRO [Computer Software]. 2016: Available from: https://www.elsevier.com/solutions/macro 